\title{
Epilepsy: Age and Gender Aspects
}

\author{
Anna A Voitiuk* \\ Department of Neurology and Child Neurology, Ukraine
}

*Corresponding author: Anna A Voitiuk, Department of Neurology and Child Neurology, Kharkiv Medical Academy of Postgraduate Education, Kharkiv, Ukraine.

Received Date: April 23, 2019

Published Date: May 29, 2019

\begin{abstract}
The relevance of specific gender issues has been steadily increasing in recent years. The incidence of epilepsy among men and women in the population is approximately the same, but men are somewhat dominated in the adult period of life. The physiological, psychological and hormonal differences between a woman and a man cause a differentiated approach to the treatment of epilepsy depending on the sex of the patient. Thus, the problems associated with the reproductive health of men and women suffering from epilepsy accompany them throughout their lives, reducing the quality of life.
\end{abstract}

Keywords: Epilepsy; Quality of life; Men; Women; Gender peculiarities

\section{Review}

Currently, according to the World Health Organization, there are more than 65 million people with epilepsy in the world. The prevalence of epilepsy in developed countries is 5-10 cases per 1000 population [1]. According to the results of demographic studies conducted in developed countries, the incidence of epilepsy varies from 0.28 to 0.53 per 1000 population [2]. In the CIS countries, the prevalence of the disease varies from 0.96 to 10 cases per 1,000 population [1]. The prevalence of epilepsy ranges from 50 to 73 cases per 100,000 population in various areas in Ukraine (an average of 73.9 per 100,000). The incidence is $50-70$ cases per 100000 population per year, the prevalence is 5-10 per 1000 population. In addition, the average incidence in men (53.7 per 100,000$)$ is slightly higher than in women $(46.3$ per 100,000$)$ [3]. Epilepsy is one of the most frequent and serious neurological diseases and ranks third among organic brain diseases.

This disease places a burden on the patient, his family and society as a whole. Epilepsy affects social functioning, limiting employment opportunities and education, affects the patient's social circle, and increases the risk of death. The burden of epilepsy is associated with many factors, including the unpredictability of seizures and stigmatization of patients [4].

According to the consensus of the International League against epilepsy (International League Against Epilepsy, ILAE) and International Bureau for epilepsy (International Bureau for Epilepsy, IBE), epilepsy is a disease of the brain defined by any of the following conditions:

1. At least two unprovoked (or reflex) seizures occurring $>24$ h apart

2. One unprovoked (or reflex) seizure and a probability of further seizures similar to the general recurrence risk (at least $60 \%$ ) after two unprovoked seizures, occurring over the next 10 years

3. Diagnosis of an epilepsy syndrome [5].

Epilepsy is a separate problem for each sex, which requires gender-specific management approaches and patient treatment strategies. If we take into account differences in the structure of epilepsy depending on gender, then a significantly higher percentage of symptomatic post-traumatic epilepsy and alcohol dependence is characteristic of males. Epilepsy with myoclonic-astatic seizures (Doose syndrome) boys suffer 2 times more often, focal seizures with hypermotor automatisms are 2 times more common in men. [4] Sudden death syndrome with epilepsy (SUDEP) is also more common in men [6]. However, there are epileptic syndromes that are more common in women: pediatric absence epilepsy, typical early onset absences, photosensitive forms of epilepsy, juvenile 
myoclonic epilepsy, catamenial epilepsy [7]. Before the age of 10, pseudo-epileptic paroxysms are equally common in boys and girls, after 10 years are more often in girls. In adulthood in $60-80 \%$ of cases women suffer from pseudo-epileptic paroxysms [2].

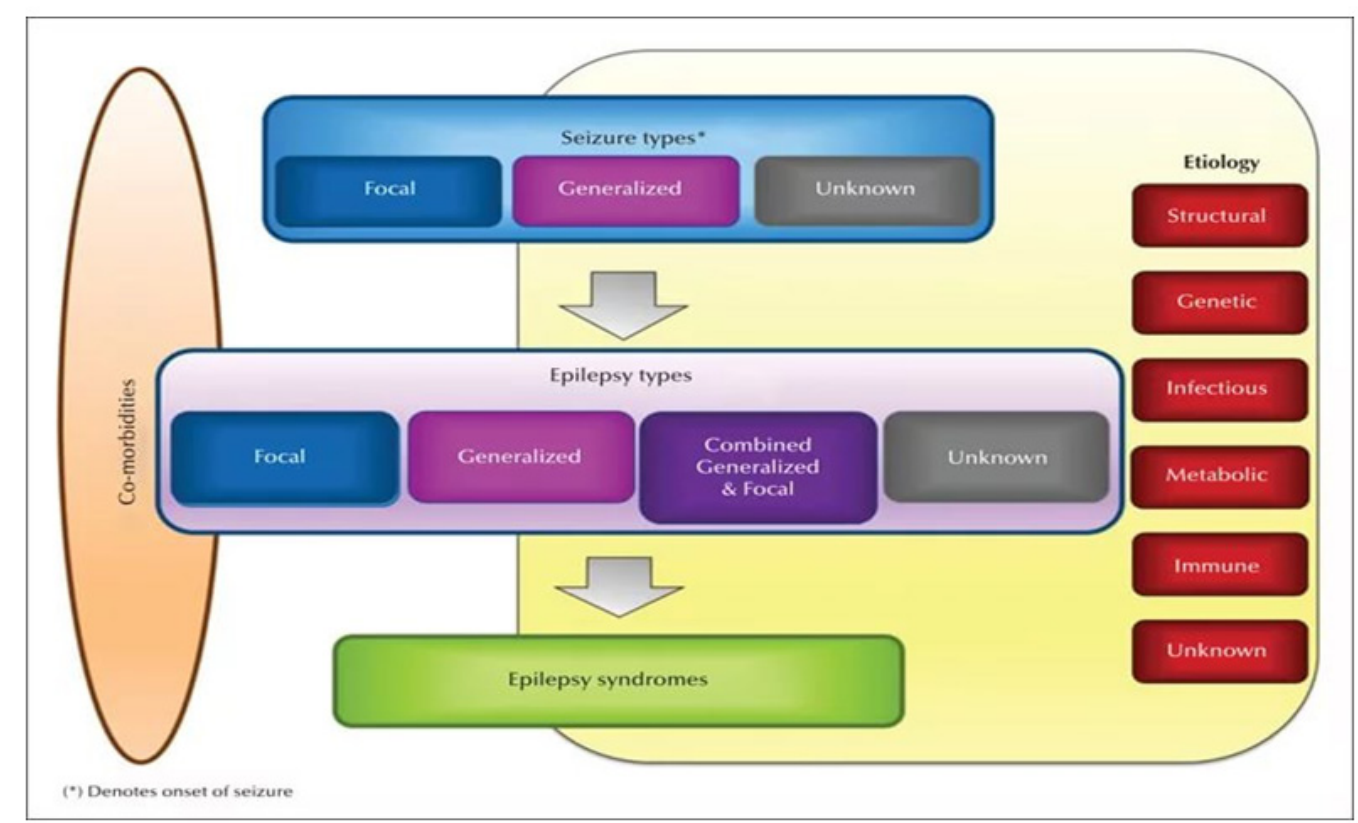

Figure 1: Framework for epilepsy classification.

In the pubertal period and up to 30 years, and also over the age of 70 years, the prevalence of epilepsy in women prevails. The predominance of patients with epilepsy in women over the age of 70 is associated not only with absolute dominance in the population of this age of women, but also with the manifestation and progression of vascular pathology [7]. At puberty, young men are much more likely to work in their free time, and such work can lead to sleep deprivation and provoke epileptic seizures $[8,9]$.

Epilepsy and antiepileptic drugs (AEDs) can comprehensively affect the reproductive system, leading to a decrease in fertility in both women and men, as well as causing various endocrine disorders: hyperandrogenation, deviations in the concentration of luteinizing hormone, follicle stimulating hormone, etc. In men, epilepsy and AEDs, as in women, cause dysfunction of the hypothalamic-pituitary-sexual system at all its levels. Epileptic seizures are often associated with hormonal disorders, causing the release of hypothalamic and pituitary hormones. Also, some AEDs can alter the level of sex hormones, as well as induce their effects [10]. Interictal increase of prolactin is observed in men and women, regardless of the conduct of anti-epileptic therapy. An epileptic seizure may cause an increase in the level of prolactin, which reaches a maximum after 20 minutes and persists for 1 hour after the attack. In addition, a high level of prolactin is one of the most common causes of impotence in men without epilepsy [11].

Changes in the level of luteinizing hormone (LH) also contribute to sexual impairment. It was noted that a low level of LH corresponds to a low level of sexual desire in women with temporal epilepsy $[12,13]$. Although the overall level of steroid hormones is not altered in most patients with epilepsy, the concentration of free hormone fractions, which are biologically active components, can be reduced [14]. Low testosterone levels were associated with impaired sexual desire and activation in men with epilepsy. Sexual dysfunction may be due to ictal changes in neurotransmitters, including GABA, endorphins and serotonin. Postictal increase in the level of GABA and endorphins is accompanied by prolonged neural depression [15]. Finally, the treatment of AEDs can contribute to the emergence of sexual dysfunction, since AEDs can alter the concentration of steroid sex hormones and, therefore, affect mood, behavior, and sexual desire.

In modern literature there is widespread debate about the different impact of AEDs on reproductive health. Valproates are considered to have the most adverse effects on the reproductive system, the smallest are the new generation AEDs. Treatment oxcarbazepine, as well as treatment with lamotrigine was not associated with reproductive disturbances. Hormonal and endocrine disorders in the use of levetiracetam have not been described [16]. Gender orientation (masculine, feminine) has different effects on the strategy of human behavior, their social functioning, adaptation. In this regard, the prevalence, course of many diseases (pathogenesis, risk factors), the response to treatment will depend on the gender and gender characteristics of the individual. The nature of psychological and cognitive impairment depends on the duration of persistent seizures, the form of the disease. The psyche of a patient with epilepsy reflects the influence of a complex of factors, namely, epileptogenic lesion, a specifically altered function of neurons and neural ensembles, localization of the lesion, the nature and frequency of attacks, premorbid States of the individual, the reaction of the individual to the disease, violations in the microsocial sphere, constant frustration, feelings of threat of violation in personal and social life [17]. 
The connection between the organic and the psychic in epilepsy is always refracted by the reactions of the brain itself - its individual genetic Constitution and the psychological framework of the personality, as well as psychological mechanisms of protection that arise when the patient interacts with the surrounding society; this manifests the relationship of the endogenous and social. Epileptic focus, forcing your mode of operation to the whole brain or a significant part thereof, will disrupt its function, which may manifest itself in various behavioral and other disorders occurring in combination with epileptic seizures, and without them. Establishing a diagnosis of epilepsy is a label that leads to a number of limitations in everyday life and professional activities. Ultimately, this causes the patient's gradual frustration, accompanied by depression and other mental disorders, not to mention the role of the seizure itself as a constant threat to the health of the patient and his social well-being.

The problems of people suffering from epilepsy are related not only to the clinical manifestations of this disease, but also to the attitude of patients to their disease, to the need to constantly take drugs, as well as to the attitude of society towards them, which does not always reasonably limit their desire for self-realization and social activity. Interaction of social stressors leads to failure of adaptation at biological, psychological and social levels.

Epilepsy, being initially a neurological disease, is an example of an interdisciplinary problem. Epileptic seizures, which are considered as typical neurological phenomena, lead in most patients to psychopathological phenomena (affective disorders, personality changes, mnestic-intellectual decline, epileptic psychoses), which eventually begin to be no less important for patients than the initial epileptic seizures. Here it is necessary to point out the importance of stigmatization in patients with epilepsy, caused not so much by the presence of seizures as by the diagnosis of "epilepsy" and related problems in public life.

\section{Conclusion}

Modern principles of treatment of epilepsy imply not only a quantitative indicator - a decrease in the number of attacks with the achievement of drug, and ideally, clinical remission, but also a qualitative improvement in the patient's life. The quality of life in epilepsy is closely related to the efficacy, tolerability and safety of antiepileptic therapy. Taking into account the negative impact of this disease on the quality of life of the patient, the assessment and improvement of the quality of life is recognized as an important component of the treatment program for patients with epilepsy. The quality of life of patients with epilepsy implies a socially active lifestyle - education, work, family and much more.

\section{Acknowledgement}

None.

\section{Conflict of Interest}

No conflict of interest.

\section{References}

1. Banerjee PN (2009) The descriptive epidemiology of epilepsy. In: PN Banerjee, D Filippi, W Clen Hauser (Eds.), Epilepsy Res 85: 31-45.

2. Wicks P, Fountain NB (2012) Patient assessment of physician performance of epilepsy quality-of-care measures. Neurol Clin Pract 2(4): 335-342.

3. Main Department of Statistics of the Ministry of Health in Ukraine [Electron resource]. - Mode of access: http://www.moz.gov.ua

4. Gusev EI, Geht AB (2013) Epilepsy: basic, clinical and social aspects. Moscow: APKIPPRO, Russia, p. 874.

5. Fisher RS, Acevedo C, Arzimanoglou A, Bogacz A, Cross JH, et al. (2014) A practical clinical definition of epilepsy. Epilepsia 55(4): 475-482.

6. Duncan S (2011) Epilepsy services: man up Epilepsy professional 22: 11-15.

7. Charles VA (2010) Epilepsy in children and adult women and men. Moscow: Medicine, Russia: 720.

8. Sare G, Rawnsley M, Stoneman A, Duncan S (2007) Men with epilepsy the lost tribe? Results of a nationwide survey of men with epilepsy living in the UK. Seizure 16 (5): 384-396.

9. Moshe S, Shilo M, Chodick G, Yagev Y, Blatt I, et al. (2008) Occurrence of seizures in association with work related stress in young male army recruits. Epilepsia 49: 1451-1456.

10. Bauer J, Blumenthal S, Reuber M, Stoffel-Wagner B (2004) Epilepsy syndrome, focus location, and treatment choice affect testicular function in men with epilepsy. Neurology 62: 243-246.

11. Rodin E, Subramanian M, Gilroy J (1984) Investigation of sex hormones in mate epileptic patients. Epilepsia 25: 690-694.

12. Herzog AG, Seibel MM, Schomer DL, Vaitukaitis JL, Geschwind N (1986) Reproductive endocrine disorders in men with partial seizures of temporal lobe origin. Arch Neurol 43: 347-350.

13. Herzog AG, Seibel MM, Schomer DL, Vaitukaitis JL, Geschwind N (1986) Reproductive endocrine disorders in women with partial seizures of temporal lobe origin. Arch Neurol 43: 341-346.

14. Dana Haeri J, Oxley J, A Richens (1982) Reduction of free testosterone by antiepileptic drugs. British Medical Journal 284: 85-86.

15. Paredes RG, Manero MC, Haller AE, Alvarado R, Agmo A (1992) Sexual behavior enhances postictal behavioral depression in kindled rats: opiod involvement. Behavioral Brain Res 52(2): 175-182.

16. Herzog AG1, Drislane FW, Schomer DL, Pennell PB, Bromfield EB, et al. (2004) Differential effects of antiepileptic drugs on sexual function and reproductive hormones in men with epilepsy: interim analysis of a comparison between lamotrigine and enzyme-inducing antiepileptic drugs. Epilepsia 45(7): 764-768.

17. Tokareva NG, Zheleznova EV (2015) Features of medico-social rehabilitation of patients with epilepsy. E research and educational bullet in «Health and education in the twenty-first century 17(5): 27-29. 